\title{
Chronic Myelomonocytic Leukemia: Current Classification, Diagnosis and Risk Stratification
}

\author{
Nahla A M Hamed* \\ Professor of Hematology, Alexandria University, Egypt
}

Submission: April 30, 2017; Published: May 10, 2017

"Correspondence Address: Nahla A M Hamed, Professor of Hematology, Faculty of Medicine, Alexandria University, Egypt, Email: drhamedn@hotmail.com

\begin{abstract}
CMML is a clonal malignant myeloid disorder sharing features of myelodysplastic syndromes and chronic myelo proliferative neoplasms. The 2016 revision to the WHO classification of myeloid neoplasms has recommended distinction between MP-CMML (WBC count $\geq 13 \times 10^{9} / \mathrm{L}$ ) and the MD-CMML (WBC $<13 \times 10^{9} / \mathrm{L}$ ). This sub classification might not provide prognostic value. FAB sub classification has shown a more precise prognostication with 3 blast-based groupings: CMML-0; CMML-1 and CMML-2. Gene mutations involving TET2 (w60\%), SRSF2 (w50\%), ASXL1 (w40\%), and RAS (KRAS and NRAS w20\%-30\%) are frequent. Nonsense and frameshift ASXL1 mutations are the only mutations that have an independent negative prognostic effect on overall survival. Patients presenting with a clinical phenotype of CMML with eosinophilia, should be assessed for the $t(5 ; 12)(q 31-q 32 ; p 13)$, giving rise to the ETV6(TEL)-PDGFRB fusion oncogene.
\end{abstract}

Abbreviations: CMML: Chronic Myelomonocytic Leukemia; WBC: White Blood Cell; CPSS: CMML-Specific Prognostic Scoring System; GMDAPS: Global MD Anderson Prognostic Scoring System; MMM: Molecular Mayo Model; GFM: Groupe Français des Myélodysplasies model; GM-CSF: Granulocyte-Macrophage Colony Stimulating Factor; PSTAT5: phosphorylated Signal Transducer and Activator of Transcription 5; AML: Acute Myeloid Leukemia; MP-CMML: CMML "proliferative type"; MD-CMML: CMML “dysplastic type"; ESAs: Erythropoiesis-Stimulating Agents; TIF1g: Transcription-Intermediary Factor-1 gene; UPD: Uniparentaldisomy; KPS: Karnofsky performance status

\section{Introduction}

CMML is a clonal malignant myeloid disorder sharing features of myelodysplastic syndromes and chronic myeloproliferative neoplasms. It is characterized by peripheral blood monocytosis, dysplastic features in at least 1 hematopoietic cell line and increased risk of progression to AML [1]. CMML has a male preponderance (1.5-3.0:1) [2]. The median age at diagnosis of CMML is about 70 years [3]. The exact incidence is unknown but is approximated at 4 cases per 100,000 persons per year [2].Therapy-related CMML cases have been described, and are associated with worse prognosis than de novo CMML [3]. The pathophysiology of CMML is still not fully understood. Microenvironmental influences, particularly angiogenesis, play a special role in the development and maintenance of CMML [3].

\section{WHO Sub-Classification}

Due to the discovery of molecular and clinical differences particularly those related to RAS/MAPK signaling pathways aberrancies [4], the 2016 revision to the WHO classification of myeloid neoplasms has recommended distinction between MPCMML (WBC count $\geq 13 \times 10^{9} / \mathrm{L}$ ) and the MD-CMML (WBC $<13$ x $10^{9} / \mathrm{L}$ ). There is apparent difference in survival between the two subtypes probably accounted for by the higher prevalence of leukocytosis/ monocytosis and of ASXL1 mutations in MPCMML. However, this subclassification might not provide additional prognostic value [5].

\section{FAB Sub-Classification}

This has shown a more precise prognostication with 3 blastbased groupings. CMML-0, a category for cases with $<2 \%$ blasts in $\mathrm{PB}$ and $<5 \%$ blasts in BM; CMML-1 for cases with $2 \%$ to $4 \%$ blasts in $\mathrm{PB}$ and/or $5 \%$ to $9 \%$ blasts in $\mathrm{BM}$; and CMML-2 for cases with $5 \%$ to $19 \%$ blasts in $\mathrm{PB}, 10 \%$ to $19 \%$ in BM, and/or when any Auer rods are present [6].

The clinical presentations represent the full spectrum of both MDS and MPN [3]. In most reports, patients with MP-CMML were older than patients with MD-CMML, but the differences were not statistically significant [2]. Furthermore, male predominance was observed more frequently in patients with MD-CMML [3]. SRSF2 mutations are associated with increasing age, less pronounced anemia, and a diploid karyotype [2]. Patients with an MDS phenotype tend to present with PB cytopenias, effort intolerance, easy bruising, recurrent infections, and transfusion 


\section{Cancer Therapy \& Oncology International Journal}

dependence. Those with an MPN phenotype tend to present with leukocytosis, monocytosis, hepatomegaly, splenomegaly, and features of myeloproliferation such as fatigue, night sweats, symptoms from organomegaly, bone pain, weight loss, and cachexia. Some reports have described leukemia cutis as an initial manifestation [2]or heralding transformation to AML [3]. Rates of leukemic transformation in most studies quote an incidence of $15-20 \%$ [6]. Rarely, patients directly present with blast phase disease [2].

\section{Diagnosis of CMML}

It requires both the presence of persistent $\mathrm{PB}$ monocytosis $\geq 1 \times 10^{9} / \mathrm{L}$ and monocytes accounting for $\geq 10 \%$ of the WBC differential count [4], less than $20 \%$ blasts and promonocytes in the $\mathrm{PB}$ and $\mathrm{BM}$, and dysplasia involving one or more myeloid lineages [2]. BCR-ABL1 rearrangement should be excluded and PDGFRA, PDGFRB, FGFR1 rearrangements orPCM1-JAK2 fusions excluded if eosinophilia is present [7,8]. If myelodysplasia is absent or minimal, the diagnosis of CMML can still be made if the other requirements are met and an acquired clonal or molecular genetic abnormality is present in the hematopoietic cells or ifthe monocytosis has persisted for at least 3 months and other causes of monocytosis have been excluded [2].

\section{Investigations}

There is no single finding pathognomonic of CMML diagnosis [6]. CMML patients have an increase in the fraction of classical monocytes (CD14 7 /CD16- [2]. In some cases, eosinophilia may present as a part of the CMML disease process [3]. It is important to exclude reactive nonmalignant causes of monocytosis such as tuberculosis, chronic fungal infections, subacute bacterial endocarditis, viral, and protozoal infections (leishmaniasis); connective tissue disorders, such as systemic lupus erythematosus and sarcoidosis, and lipid storage disorders. The recovery phase of an acute infection (usually viral) or bone marrow regeneration post chemotherapy is commonly associated with monocytosis [6]. Clonal monocytosis is often persistent and is associated with hematopoietic disorders such as juvenile myelomonocytic leukemia, primary myelofibrosis, AML with monocytic differentiation [2] and chronic myeloid leukemia. PDGFR rearranged myeloid neoplasms can be associated with monocytosis and BM dysplasia [6].

Bone marrow biopsies are often hypercellular with granulocytic hyperplasia and dysplasia. Monocytic proliferation is often difficult to appreciate and their identification using immunohistochemical studies are recommended [6]. Almost $80 \%$ of patients will have micromegakaryocytes with abnormal nuclear contours and lobations, and $30 \%$ of patients have an increase in BM reticulin fibrosis. Nodules composed of mature plasmacytoid dendritic cells are present in $20 \%$ of patients [2].

On immunophenotyping, the abnormal BM cells often express myelomonocytic antigens such as CD13 and CD33. There may be variable expression of CD14, CD68, and CD64, markers of aberrant expression including CD2, CD15, and CD56 or decreased expression of CD14, CD13, HLA-DR, CD64, or CD36. The presence of myeloblasts can be detected by CD34 expression. The most reliable markers on immunohistochemistry include CD68R and CD163 [2].

Cytogenetic abnormalities are present in only $25 \%$ to $30 \%$ of the patients, with trisomy 8 , loss of $\mathrm{Y}$ chromosome, abnormalities of chromosome 7, and complex karyotypes are the most frequently found [1]. Patients presenting with a clinical phenotype of CMML with eosinophilia, should be assessed for the $t(5 ; 12)$ (q31-q32;p13), giving rise to the ETV6(TEL)-PDGFRB fusion oncogene [6].

Gene mutations involving TET2 (w60\%), SRSF2 (w50\%), ASXL1 (w40\%), and RAS (KRAS and NRAS w20\%-30\%) are frequent [2]. Nonsense and frame shift ASXL1 mutations are the only mutations identified to have an independent negative prognostic effect on overall survival [2]. The presence of clonal TET2 mutations (in the absence of clonal ASXL1 mutations) have a favorable impact on OS, although, the reason for this association is unclear [6]. SRSF2 mutations have not been found to have an independent prognostic impact on either OS or LFS [2].

SETBP1gene mutations have been reported recently to have an unfavorable prognosis in CMML patients. SETBP1 mutations have been suggested to be potential drivers of other mutations that lead to poor prognosis. However, the statistically significant association between SETBP1 with other mutations is controversial [8]. Although ASXL1 and SETBP1 mutations have adversely affect survival of untreated CMML patients, their impact on hypo methylating therapy is unclear at this time [6]. Other signal pathway and transcription factor mutations in CMML include JAK2V617F (w10\%-15\%), RUNX1 (w15\%), and CBL (w10\%-20\%) [2]. UPD results from segmental DNA recombination during mitosis, was a common event in MDS/ MPN (35\%), especially in CMML patients [3].

\section{CMML Prognostic Models}

Seven clinical prognostic models, not incorporating ASXL1 mutational status (IPSS, R-IPSS, MDAPS, Global MDAPS, Dusseldorf, CPSS, and Mayo model) were valid with comparable performance, but were vulnerable to upstaging [6]. CPSS, relies on the WHO sub classification, the FAB sub classification, CMML-specific cytogenetic classification (low risk $=$ normal and isolated $-Y$; intermediate risk $=$ all other abnormalities not included in low or high risk; and high risk = trisomy 8, complex, and chromosome 7 abnormalities), and erythrocyte transfusion dependency. One point was accorded for each variable, with the exception of high-risk cyto genetics which earned 2 points. Four risk categories were determined: low ( 0 points), intermediate- 1 (1 point), intermediate-2 (2-3 points), and high (4-5points). Median OS was $72,31,13$, and 5 months respectively for each of the categories. Another recent useful risk model, the GMDAPS, was proposed for CMML patients, regardless of specific 
disease type, prior therapy, or duration of disease. G-MDAPS is based on performance status, age, platelet count, hemoglobin value, percentage bone marrow blasts, WBC count, karyotype, and prior transfusion. This model identified 4 prognostic groups with median survivals of 54 months (low), 25 months (intermediate-1), 14 months (intermediate-2), and 6 months (high), respectively. A refined MDAPS score, more specific for CMML patients, was recently proposed [3].

Two molecularly integrated prognostic models (inclusive of clinical parameters and presence or absence of the ASXL1 mutations) include the MMM and the GFM. The GFM model segregates patients into 3 groups based on: age $>65$ years, WBC $>15 \times 10^{9} / \mathrm{L}$, anemia, platelets $<100 \times 10^{9} / \mathrm{L}$, and ASXL1 mutation status, with respective median survivals of 56 months (low), 27.4 months (intermediate), and 9.2 months (high). The MMM is based on ASXL1 mutational status, absolute monocyte count $>10 \times 10^{9} / \mathrm{L}$, hemoglobin $<10 \mathrm{~g} / \mathrm{dL}$, platelets $<100 \times 10$

$/ \mathrm{L}$ and circulating immature myeloid cells. This model stratifies patients into four groups; high ( $\geq 3$ risk factors), intermediate- 2 ( 2 risk factors), intermediate-1 (1 risk factor) and low (no risk factors), with median survivals of 16, 31, 59, and 97 months, respectively. In multivariable analysis, ASXL1 mutations were independently predictive of shortened OS [6].

\section{Treatment}

\section{Iron chelation therapy}

Increased serum ferritin levels in CMML can be secondary to transfusion dependence, cancer-associated inflammation, and liver disease. However, routine use of iron chelation therapy in CMML cannot be recommended until its efficacy is established [2].

\section{The use of ESAs and transfusional supportive care}

Response rates in lower-risk MDS/CMML patients range from $30 \%$ to $60 \%$, with a median duration of response of approximately 24 months. Most responses to ESA occur within 8 weeks of treatment. Parameters predictive of ESA response include low transfusion burden $(<2 \mathrm{U} / \mathrm{mo})$, use of a fixed-dose vs weight-based erythropoietin regimen, shorter time from diagnosis to treatment initiation, and a lower baseline serum erythropoietin level ( $<500 \mathrm{IU} / \mathrm{L}$ ). ESA should be used cautiously in MP-CMML patients given the inherent risk for spontaneous splenic rupture [2].

\section{Cytoreductive agents and epigenetic modifiers}

Hydroxyurea and other cytoreductive agents have been used to control MPN-like features in Lower risk CMML patients, while epigenetic modifiers such as hypomethylating agents have been used for MDS-like features [2]. Standard induction chemotherapy should be considered for all eligible patients who develop blast transformation [6]. Hypomethylating agents are associated with overall response rates of approximately 30-40\% (range from $25-70 \%$ ) [6], with complete remission rates of about15\% [2] and median OS ranged from 12 to 37 months. These responses are generally not sustained and survival after loss of response in these patients is often dismal [6].

The presence of an elevated WBC count ( $\left.>13 \times 10^{9} / \mathrm{L}\right)$, palpable splenomegaly and increased bone marrow blast percentage $(>10 \%)$ are associated with a worse survival on hypomethylating agents [6] while an absolute monocyte count $<10 \times 10^{9} / \mathrm{L}$, and peripheral blood blasts $<5 \%$ at the start of hypomethylating therapy was associated with improved OS [6]. In CMML patients aged $<65$ years, the presence of clonal TET2 mutation in the absence of ASXL1 mutations has been associated with response to 5 -azacitidine and decitabine [6]. A minimum of 4 cycles are generally required before response assessment can be made [2].

Mutations in ASXL1, NRAS, KRAS, CBL, FLT3, and JAK2 genes, and hyper methylation of the promoter of the tumor suppressor gene-TIF1g did not predict response or survival in $39 \mathrm{CMML}$ patients treated with decitabine. However, lower CJUN and CMYB gene expression levels independently predicted improved OS [6].

Role of allogeneic stem cell transplant in CMML remains controversial [3]. Older patients with a high HCT comorbidity index do not benefit from SCT, and are best suited for clinical trials while it is the treatment of choice for younger patients with higher risk disease as determined by newer prognostic models incorporating molecular aberrations [6]. Myeloablative regimens are preferred over reduced-intensity conditioning because of the higher relapse rates with the latter approach. Cyto reductive therapy or HMAs is often considered before HSCT in patients with increased BM blasts (CMML-2) or before reduced-intensity conditioning [2]. Higher CPSS score at time of transplantation; lower KPS and a bone marrow graft are associated with inferior survival after HCT [9]. Complications of HSCT including non relapse mortality and acute and chronic GVHD and post-transplant disease relapse limit generalized applicability of this treatment strategy [6].

Given the relatively poor response to these drug agents and the inherent risks associated with hematopoietic stem cell transplant, newer drugs exploiting molecular and epigenetic abnormalities in CMML are being developed. The creation of CMML-specific response criteria is a much needed step to improve clinical outcomes [2].

Investigational agents used for treatment of CMML include topoisomerase I inhibitors (topotecan), deoxycytidine analog (sapacitabine), a second-generation nucleoside analog (clofarabine), tyrosine kinase inhibitors, the immunomodulator thalidomide/lenalidomide, all-trans retinoic acid, histone deacetylase inhibitors, JAK2 inhibitors ruxolitinib, and the RASactivating enzyme farnesyltransferase inhibitors (lonafarnib, tipifarnib) [3]. Additionally, targeted anti-GM-CSF monoclonal antibody therapy is being developed owing to the inherent, demonstrable, GM-CSF dependent pSTAT5 sensitivity in CMML patients (NCT02546284; http://www.clinicaltrials.gov) [6]. 


\section{Cancer Therapy \& Oncology International Journal}

\section{Reference}

1. Sanz GF (2017) A lot to learn about allogeneic hematopoietic cell transplantation for chronic myelomonocytic. Biol Blood Marrow Transplant 23(5): 713-714.

2. Patnaik MM, Tefferi A (2016) Chronic myelomonocytic leukemia: focus on clinical practice. Mayo Clin Proc 91(2): 259-272.

3. Bentona CB, Nazhab A, Pemmarajuc N, Garcia-Maneroc G (2015) Chronic myelomonocytic leukemia: forefront of the field in 2015. Crit Rev Oncol Hematol 95(2): 222-242.

4. Arber DA, Orazi A, Hasserjian R, Thiele J, Borowitz MJ, et al. (2016) The 2016 revision to the World Health Organization classification of myeloid neoplasms and acute leukemia. Blood 127(20): 2391-2405.

5. Patnaik MM, Lasho TL, Frinke C, Howard MT, Hanson CA, et al. (2016) Proliferative versus dysplastic chronic myelomonocytic leukemia: molecular and prognostic correlates. Blood 128(22): 1987.
6. Tefferi A, Patnaik M (2016) Chronic myelomonocytic leukemia: 2016 update on diagnosis, risk stratification and management. Am J Hematol 91: 632-642.

7. Nakata Y, Ueda T, Nagamachi A, Yamasaki N, Ikeda K, et al. (2016) Acquired expression of $\mathrm{Cbl}^{0367 \mathrm{P}}$ in mice induces dysplastic myelopoiesis mimicking chronic myelomonocytic leukemia. Blood 129(15): 21482160.

8. Shou L-H, Cao D, Dong X-H, Fang Q, Wu Y, et al. (2017) Prognostic significance of SETBP1 mutations in myelodysplastic syndromes, chronic myelomonocytic leukemia and chronic neutrophilic leukemia: A meta-analysis. PLoS One 12(2): 0171608.

9. Liu HD, Ahn KW, Hu ZH, Hamadani M, Nishihori T, et al. (2017) Allogeneic hematopoietic cell transplantation for adult chronic myelomonocytic leukemia. Biol Blood Marrow Transplant 23(5): 767 775 .

Your next submission with Juniper Publishers will reach you the below assets

- Quality Editorial service

- Swift Peer Review

- Reprints availability

- E-prints Service

- Manuscript Podcast for convenient understanding

- Global attainment for your research

- Manuscript accessibility in different formats

( Pdf, E-pub, Full Text, Audio)

- Unceasing customer service

Track the below URL for one-step submission https://juniperpublishers.com/online-submission.php 